\title{
Féeries
}

Études sur le conte merveilleux, XVII $-\mathrm{XIX}{ }^{\mathrm{e}}$ siècle

16 | 2020

Le conte, les mythes antiques, la Bible

\section{La souveraine du lac gelé : variantes et altérations du mythe d'Hérodiade dans les croyances populaires européennes}

The sovereign of the frozen lake: variants and alterations of the myth of Herodias in European popular beliefs

\section{Stamatios Zochios}

\section{OpenEdition}

Journals

Édition électronique

URL : https://journals.openedition.org/feeries/2498

DOI : $10.4000 /$ feeries. 2498

ISSN : 1957-7753

Éditeur

UGA Éditions/Université Grenoble Alpes

Édition imprimée

ISBN : 978-2-37747-194-2

ISSN : $1766-2842$

\section{Référence électronique}

Stamatios Zochios, «La souveraine du lac gelé : variantes et altérations du mythe d'Hérodiade dans les croyances populaires européennes », Féeries [En ligne], 16 | 2020, mis en ligne le 11 novembre 2020, consulté le 10 décembre 2021. URL : http://journals.openedition.org/feeries/2498 ; DOI : https:// doi.org/10.4000/feeries.2498

Ce document a été généré automatiquement le 10 décembre 2021.

(c) Féeries 


\title{
La souveraine du lac gelé : variantes et altérations du mythe d'Hérodiade dans les croyances populaires européennes
}

\author{
The sovereign of the frozen lake: variants and alterations of the myth of \\ Herodias in European popular beliefs
}

Stamatios Zochios

\section{La vie et le nom énigmatique d'Hérodiade}

$1 \quad$ L'une des figures bibliques les plus connues et à la fois les plus intrigantes et détestées dont la vie infâme a nourri les arts à maintes reprises est Hérodiade ${ }^{1}$. Considérée comme une représentante principale du " pouvoir des femmes ${ }^{2}$ ", ce topos artistique du Moyen Âge et de la Renaissance d'après lequel l'inversion de la hiérarchie sexuelle rend la femme dominante, elle a inspiré entre autres Titien, Le Caravage, Delaroche, Moreau, Heine, Flaubert, Wilde, Massenet, Strauss, Hindemith.

2 Qui est vraiment Hérodiade, cette princesse juive descendante des Hasmonéens? De son vrai nom Hérodias (elle est nommée Hérodiade dans la tradition française), née à Jérusalem vers l'an 15 av. J.-C. et morte après l'an 39, est la fille d'Aristobule IV (vers $35 /+7)$, assassiné par son père, le roi de Judée, Hérode ${ }^{\text {er }}$ le Grand $(-73 /+4)$, qui voulait consolider son pouvoir ${ }^{3}$. Hérodiade avait alors onze ans. Elle épouse le frère de son père, Hérode Boëthos (vers -27 / vers +34 ), avec lequel elle habite probablement à

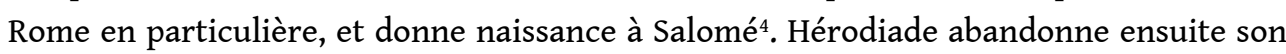
époux Hérode Boëthos pour vivre avec un autre frère de son père, Hérode Antipas (-21 / +39), un tétrarque juif qui abandonne également son épouse. Ces évènements déclenchent la colère du prédicateur juif Yohanan (dit Jean le Baptiste), qui dénonce les turpitudes d'Hérodiade. 
Par la suite, Hérode Antipas, comme le rapporte l'évangéliste Luc, «étant repris par [Jean] au sujet d'Hérodiade, [...] et de tout le mal qu'il avait fait, il ajouta ce crime à tous les autres, et enferma Jean en prison " (Luc 3,19). Lors de la fête de l'anniversaire d'Hérode Antipas, où Salomé danse et lui "pl[aît ainsi qu']à ses convives", il lui demande ce qu'il peut lui offrir comme récompense (Marc 6,23). Salomé, exhortée par sa mère Hérodiade, demande la tête de Jean présentée sur un plateau. Bien qu'Hérode Antipas craigne la popularité de Jean (Matthieu 14,5), obligé de tenir sa promesse et attristé, il ordonne sa décapitation vers l'an 28 (Matthieu 14,9-10).

4 Ainsi, en raison de tout cela, Hérodiade a été tenue pour responsable de la mort de Jean le Baptiste et a provoqué la haine du monde chrétien. Il faut pourtant noter que l'utilisation du nom Hérodiade pose problème parce qu'il correspond à la fois à Hérodias et à Salomé ${ }^{5}$. Cette ambiguïté onomastique tiendrait au fait que, selon Waldemar Kloss, dans la littérature hellénistique, les patronymes étaient employés de manière très souple ${ }^{6}$. La détestation de ces deux femmes, Hérodias et Salomé, s'est transformée graduellement, dans la conscience collective des Chrétiens, en un personnage diabolique sous le nom indistinct d'Hérodiade. Cette figure a acquis des pouvoirs surnaturels dans l'imaginaire populaire. Ainsi, comme Mireille Dottin-Orsini le note, « réduites ensuite à l'existence souterraine des dieux mis à l'index, mère et fille connaissent des avatars populaires ${ }^{7} »$.

\section{La souveraine des sorcières}

5 Cette procédure de diabolisation était déjà accomplie à la fin du $\mathrm{I}^{\mathrm{er}}$ millénaire. Comme signalé dans les Praeloquia (première moitié du $\mathrm{x}^{\mathrm{e}}$ siècle) de l'évêque, abbé et théologien Rathier de Véronne, les hommes impies rendent hommage à Hérodiade, la meurtrière du précurseur et baptiste du Christ, la reconnaissent comme leur souveraine, voire comme leur déesse, et dans leur lamentable démence, ils affirment qu'un tiers du monde est soumis à sa souveraineté, comme si c'était une récompense pour le meurtre du prophète 8 . Hérodiade était donc vénérée comme une souveraine maléfique. Cette tradition s'est stabilisée au cours du II $^{\mathrm{e}}$ millénaire et Hérodiade est entrée dans le panthéon démoniaque de ces divinités orchestrant les assemblées des sorcières. Initialement, son nom était associé à celui de Diane, qui était sans aucun doute considérée dans les traités médiévaux comme la divinité principale de ceux qui s'écartent de la foi chrétienne au profit du paganisme9.

L'une des premières références claires à Diane en tant que souveraine des sorcières se trouve dans le célèbre Canon Episcopi, partie du Jus Canonicum, qui date du haut Moyen Âge et qui est une source essentielle sur les vols nocturnes des sorcières. Bien que le texte ait été considéré pendant le bas Moyen Âge comme écrit au concile d'Ancyre en 314 , on le trouve pour la première fois à la fin du $\mathrm{IX}^{\mathrm{e}}$ siècle, dans le Liber de disciplinis de l'abbé Reginon de Prüm (II,364). À son tour, ce dernier ouvrage vient probablement des capitulaires carolingiens de Charles le Chauve. Par la suite, nous retrouvons le texte dans plusieurs collections de décrétales, comme celle de Burchard de Worms ( $\mathrm{xI}^{\mathrm{e}}$ siècle), d'Yves de Chartres ( $\mathrm{xl}^{\mathrm{e}}$ siècle), de Gratien ( $\mathrm{xII}^{\mathrm{e}}$ siècle), ou de Bernardin de Sienne ( $\mathrm{XIV}^{\mathrm{e}}-\mathrm{Xv}^{\mathrm{e}}$ siècles), qui tous ont critiqué les vols et les voyages nocturnes de certaines femmes. Dans l'extrait, nous trouvons plusieurs éléments liés aux divinités de la nuit, principalement Diane, et les femmes qui constituent son cortège apparaissant parfois sous la forme d'animaux ou de morts, et participant à son jeu $(l u d u s)^{10}$ : 
Il faut ajouter encore que certaines femmes scélérates retournant à Satan, séduites par les illusions et les fantasmes du démon, croient et professent que pendant les nuits, avec Diane, déesse païenne, et une innombrable tourbe de femmes, chevauchant des bêtes, elles traversent les espaces dans le calme des nuits, obéissent à ses ordres, comme à leur maîtresse absolue ${ }^{11}$.

Dans les éditions plus tardives, et plus précisément dans le Decretum de Burchard de Worms, compilé au début du $\mathrm{xI}^{\mathrm{e}}$ siècle, le nom Hérodiade est inséré comme synonyme de Diana (cum Diana paganorum dea, vel cum Herodiade). Ce passage a très probablement constitué la base des affirmations correspondantes des théologiens du Moyen Âge et de la Renaissance, comme Albert le Grand ${ }^{12}$ et Martín de Castañega ${ }^{13}$, et de nonthéologiens comme Paolo Grillandi ${ }^{14}$, Johannes Weyer ${ }^{15}$ et Jean Pic de la Mirandole ${ }^{16}$, tous utilisant le nom Hérodiade comme synonyme de Diana. De plus, l'identification d'Hérodiade à Diana a été appliquée assez tôt par les théologiens-inquisiteurs dans des affaires de sorcellerie. En 1390 par exemple, une femme accusée d'être sorcière, appelée Sibilia, confesse devant l'Inquisition milanaise qu'elle participe au jeu de Diana, qui s'appelle aussi Hérodiade ${ }^{17}$. L'assimilation des deux souveraines est devenue si courante qu'elle a donnée naissance au nom composé Herodiana. Le prédicateur franciscain du $\mathrm{XV}^{\mathrm{e}}$ siècle, Bernardin de Sienne, l'a employé comme synonyme du nom obscur Zobiana (Iobiana, Giobiana), en ajoutant que cette dernière, selon les croyances, retourne une fois par an au Jourdain pour se baptiser mais qu'elle trouve la rivière sèche ${ }^{18}$. Le nom Herodiana est resté connu longtemps, comme son utilisation par le théologien protestant Salomon Van Til à la première moitié du XVIII ${ }^{\mathrm{e}}$ siècle le prouve ${ }^{19}$.

8 L'identification d'Hérodiade avec des divinités, qui étaient plus ou moins considérées comme menant des pratiques magiques visant à assurer la fertilité et qui ont été traitées par l'Église dès le début comme hérétiques ou diaboliques, a été plus extensive et complexe. Au XV $\mathrm{XV}^{\mathrm{e}}$ siècle, le Die Himelsstraße $(\mathrm{I}, 29)$ du prieur du chapitre collégial de Sainte-Dorothée à Vienne, Stéphane de Landskrona, présente Dyana comme «une déesse païenne ou diablesse » et l'identifie à frawn Percht, frawn Hold ${ }^{20}$ et Herodyasis ${ }^{21}$. Un autre lien similaire, cette fois avec une déesse plus énigmatique, peut être trouvé chez Auger de Montfaucon, évêque de Conserans entre 1279 et 1304, exigeant " qu'aucune femme n'ose se vanter qu'elle va la nuit à cheval avec Diane, déesse des païens, ou avec Hérodiade et Bensozia ${ }^{22} »$. Les informations sur cette dernière souveraine, dont le nom signifie probablement la bonne société, à savoir les sorcières rassemblées, sont très peu nombreuses. D'après Jacques Collin de Plancy, les femmes qui allaient à une espèce de sabbat « avaient pour chef la diablesse ou fée Bensozia, à qui il fallait obéir aveuglément avec une soumission sans réserve. C'était, dit-on, la Diane des anciens Gaulois; on l'appelait aussi Nocticula, Hérodiade ou la lune. On voit, dans des manuscrits de l'église de Cousérans, que des dames au XIV siècle avaient encore le renom d'aller à cheval aux courses nocturnes de Bensozia ${ }^{23}$ ". Pour la description de Bensozia, Collin de Plancy s'est basé sur les sources citées ci-dessus, et surtout sur Auger de Montfaucon. En ce qui concerne en revanche le nom obscur Nocticula, il est fort probable que l'auteur se soit appuyé sur le Polycraticus du philosophe et historien anglais Jean de Salisbury où on lit :

On assure que la nocticole, ou une certaine Hérodiade (quale est quod nocticulam quandam vel Herodiadem), nommée aussi Maîtresse de la Nuit (noctis dominam), réunit des conseils, des assemblées nocturnes, pour célébrer des festins, des sortes d'offices aux fonctions variées, au cours desquels tantôt les mauvais sont punis et châtiés, tantôt les bons sont glorifiés. Par ailleurs, les jeunes enfants sont exposés aux lamies lors de ces cérémonies (praeterea infantes exponi lamiis). Soit ils sont 
déchirés par erreur à cause de la voracité gloutonne des lamies, et ils s'entassent, déglutis, dans leurs ventres. Soit, la présidente décide de les épargner et ils sont rejetés et déposés dans des berceaux ${ }^{24}$.

L'extrait spécifique fait longuement référence à la coutume de la célébration des déesses nocturnes. Des festins sont pratiqués, des tables sont préparées en l'honneur d'Hérodiade ${ }^{25}$, une certaine maitresse de nuit qui s'appelle aussi Nocticula - type probablement erroné de Noctiluca, «surnom donné à la lune qui brille durant la nuit ${ }^{26} »$. Claude Lecouteux traduit le nom par nocticole mais Waldemar Kloss ne le traduit pas, en indiquant que Noctiluca est « une épithète de Diana ${ }^{27}$ ». À propos de ce nom, le moine bénédictin du XVII ${ }^{e}$ siècle, Bernard de Montfaucon, note :

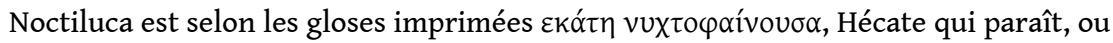
qui luit la nuit. Hécate à trois faces passait ordinairement pour Diane selon ce vers de Virgile : Tergeminamque Hecaten, tria Virginis ora Diance. Cette Noctiluca de Jean de Salisberi était donc la même que la Diane d'Auger de Montfaucon. Dans le grand manuscrit du Glossaire de cette Bibliothèque, qui a plus de neuf cent ans d'âge, on lit, Nocticula Luna : c'est une erreur manifeste, il y faut Noctiluca ; Noctiluca est donc la Lune, et la Lune est Diane comme nous avons tant de fois dit ; elle s'appelait aussi Diane Lune. Elle était censée présider à ces assemblées nocturnes, elle ou Hérodiade, qui est aussi nommée expressément dans les statuts ci-dessus ; ou enfin la Dame présidente de la nuit, par où il entend peut-être celle qui est appelée cidessus Bensozia, de laquelle je ne trouve rien dans les auteurs; c'étaient ces trois, selon Jean de Salisberi, Nocticula, Hérodiade, et la princesse de la nuit, qui convoquaient pendant la nuit des assemblées, où l'on faisait de superbes festins, où l'on voyait une grande quantité de ministres et de serviteur occuper en différents emplois $[. . .]^{28}$.
\end{abstract}

10 D'après l'explication de Bernard de Montfaucon, nous avons toutes les raisons pour identifier Hérodiade à Diana, mais aussi à Bensozia et à Noctiluca. En répétant les indications de Jean de Salisbury, Bernard de Montfaucon soutient par ailleurs que le culte de la déesse est suivi d'un spectacle horrible : les nouveau-nés et les enfants sont massacrés et dévorés par les sorcières. Le texte de Jean de Salisbury utilise pourtant le nom lamia au pluriel - lamiae - en mentionnant que les petits enfants sont exposés à ces femmes démoniaques. Cette référence rapproche la coutume de la préparation des tables et des offrandes (mensas ornare) à Hérodiade, à Diana, puis à Lamia, ici au pluriel, nom d'un personnage monstrueux de la mythologie grecque qui est devenu synonyme de sorcière pendant le Moyen Âge et la Renaissance ${ }^{29}$. Nous constatons par conséquent la liaison d'Hérodiade à une déesse païenne qui se trouvait au centre des sabbats, Diana, et ensuite aux Bensozia, Huld, Percht, Lamia, figures manifestant plus ou moins un caractère diabolique mais aussi liées à la troisième fonction dumézilienne, celle de la prospérité, assurée par des pratiques do ut des comme celle du mensas ornare.

11 Les passages cités plus haut semblent être basés sur la même tradition selon laquelle la meurtrière de Jean le Baptiste a été transformée en démon orchestrant les assemblées des sorcières. Comme nous l'avons souligné, le doute subsiste quant au fait que cette souveraine nocturne corresponde à Salomé ou à sa mère Hérodias, et aucune des sources citées ne fournit d'informations de fond sur ce qui a conduit à la transformation en démon. En revanche, le texte le plus complet et qui éclaire de nombreux points intéressants sur la mythologie d'Hérodiade - qui est ici Salomé - est le fabliau du XII ${ }^{\mathrm{e}}$ siècle, Ysengrimus, qui la décrit ainsi :

Et la vierge Pharaïlde condamnée par un saint à une peine injuste, mais les saints font ce qu'ils veulent! Hérode était célèbre à cause de cette enfant et aurait pu être heureux grâce à elle, mais un amour contrarié fit aussi le malheur de cette jeune 
fille. Cette vierge brûlait de partager la couche de Jean-Baptiste et avait fait vœu de n'appartenir à aucun autre homme. Le père, ayant découvert l'amour de sa fille, s'en irrita et fit avec cruauté décapiter le saint innocent. Désespérée, la jeune fille demanda qu'on lui apportât la tête coupée, et un serviteur du roi la lui apporta dans un plat. Elle la serre doucement entre ses bras, la baigne de larmes qu'elle désire accompagner de baisers. Mais tandis qu'elle cherche à lui prodiguer des baisers, la tête la fuit et la repousse en soufflant, et elle est emportée à travers le toit par le tourbillon que souffle le saint. Depuis, sous la forme d'un souffle, la colère de JeanBaptiste, qui se souvient trop du passé, la poursuit à travers l'espace désert du ciel. Mort, il la tourmente, et vivant, il ne l'avait pas aimée. Cependant le destin n'a pas permis qu'elle meure tout à fait: les honneurs adoucissent son deuil, le respect atténue sa peine. Le tiers de l'humanité sert cette souveraine affligée qui se repose sur les chênes et les noisetiers de la seconde partie de la nuit jusqu'au dernier chant $\mathrm{du}$ coq noir. Elle a pour nom aujourd'hui Pharaillde: elle s'appelait autrefois Hérodiade : danseuse qui n'eut d'égale ni avant elle, ni après ${ }^{30}$.

Dans ce passage, l'exceptionnelle danseuse Salomé tombant amoureuse de Jean le Baptiste s'appelle Pharaïlde. Le nom correspond à la sainte patronne de Gand. Et bien que l'identification ne soit pas évidente au premier abord, selon Carlo Ginzburg, parmi les nombreux noms qui étaient attribués à la déesse, il y avait également celui de la sainte qui, selon une légende, avait ressuscité une oie en en ramassant les os, pratique qui, pour l'historien, fait référence à des pratiques et rites magiques-extatiques ${ }^{31}$. Jeffrey Burton Russel, de son côté, signale que vers 1110 le nom Pharaïlde a été mélangé avec celui de la déesse païenne germanique Frau Huld ${ }^{32}$. Une explication beaucoup plus rationnelle est donnée par Jean Bormans, qui tire le nom Hérodiade du grec $\varepsilon \rho \alpha ́ c \omega$, " aimer ", considérant le nom Pharaillde (Ware-hild) qui signifie en tudesque "véritable amour ", comme une traduction du premier ${ }^{33}$.

Selon le fabliau, Pharaillde est condamnée, à cause de sa demande de lui apporter la tête de Jean le Baptiste, à une punition éternelle comme un autre Juif Errant: la tête de Saint Jean la fuit et la repousse en soufflant, puis la poursuit à travers l'espace désert du ciel. Pour cette raison, elle est capable de voler mais sa fuite par le toit rappelle les sorcières qui ont souvent le don de voler et d'entrer ou de sortir par la cheminée et le toit. À propos de cette capacité-punition, en Espagne on parle « de la danse aérienne à laquelle Hérodiade fut condamnée pour avoir provoqué la mort de Baptiste (de la dansa aérea á que están condenadas las Herodiadas por la muerte del Bautista $)^{34} »$.

\section{La fée dansante}

Dans cette condition posthume, selon le fabliau, Hérodiade est honorée par le tiers de l'humanité et ainsi elle se repose comme un genius loci-un esprit attaché au lieu comme par exemple une fée ou un lutin ${ }^{35}$ - sur les chênes et les noisetiers pendant la seconde partie de la nuit jusqu'au dernier chant du coq noir. Ce dernier point prouve bien sa transformation en être démoniaque qui fuit la lumière du jour. La transformation de Hérodiade en un type de fée, ou souveraine des fées, est plus claire dans les traditions sardes et roumaines. Selon Sabina Magliocco, on mentionne en Sardaigne l'existence de certains êtres appelés janas, dont le nom signifie « disciples de Diane ", les reliant directement aux légendes médiévales des esprits errants. On dit qu'ils vivent dans des tombeaux à puits néolithiques, connus sous le nom de domus de janas, «maisons des fées ", ou dans des grottes, lieux de sépultures préhistoriques ${ }^{36}$. Ce sont des tisserands experts, qui peuvent interagir avec les humains et parfois même se 
marier avec eux. Enfin les janas sardes ont pour patronne Araja ou Arada, dont le nom est une version de la médiévale italienne Hérodiade ${ }^{37}$. De manière similaire, Hérodiade participe aux coutumes de parades et de mascarades roumains, et plus précisément au căluş ${ }^{38}$. Selon Mircea Eliade ${ }^{39}$, «la patronne de la société purifiante secrète des călușarii est la "reine des fées" (Doamna Zinelor), métamorphose roumaine de Diane. Elle s'appelle également Irodiada (= Herodiada) ou Arada, deux noms célèbres parmi les sorcières d'Europe occidentale. [...] Les relations entre căluşari et zine sont paradoxalement ambivalentes : les danseuses demandent et comptent sur la protection d'Hérodiade, mais elles risquent également de devenir les victimes de sa troupe de passants, les fées ${ }^{40} »$.

\section{La fièvre d'Hérodiade}

L'apparition d'Hérodiade dans les traditions roumaines ne s'arrête pas là. Les deux versions roumaines de l'Index de livres interdits, publiés au xvII siècle, présentent les sept filles du «Roi Hérode ${ }^{41}$ » qui apparaissent comme des démons devant un Saint Sisinnios et sont vaincues par lui. Les textes ont été considérés comme des apocryphes et sont des variantes des fameuses historiolae ${ }^{42}$ byzantines relatives à Gylou ${ }^{43}$, qui ont été utilisées comme des incantations contre une série de maladies et la mort des nouveaunés. Dans les versions roumaines, les filles d'Hérode sont décrites comme des démons de la fièvre. Le motif de la décapitation de Jean le Baptiste intervient également ${ }^{44}$. Sur la même base, une autre incantation, cette fois du XVIII ${ }^{e}$ siècle, sert contre la fièvre :

Prière contre la fièvre

Parce que la miséricorde et le soutien du serviteur de Dieu (nom de la personne à se protéger) viennent de la part de Dieu. Que tu sois maudit, démon de la fièvre venant du lac et de la fièvre, avec le nom de saint Jean le Baptiste. Je te maudis, démon de la fièvre venant du lac et de la fièvre, avec les noms des saints archanges Michel et Gabriel, avec le nom du saint martyr Jean et du saint martyr Sacail Socail Socoil [...] fièvre de neuf types, au nom du Père, du Fils et du Saint-Esprit, maintenant et à jamais, amen ${ }^{45}$.

Le premier protecteur contre ces démons de la fièvre - qui sortent de l'eau - est tout d'abord le martyre prophète Jean le Baptiste dont le nom ne figure pas dans les versions grecques de Gylou, et dont l'apparition ici est justifiée parce qu'il semble que les démons de la fièvre correspondent aux filles d'Hérode. Les autres protecteurs sont les Archanges et les martyres Sacail, Socail, Socoil qui correspondent aux Sisoès, Sinès, Sinodore et Sisinnios de la version grecque de Gylou. En outre, cette incantation est apparemment liée aux versions slaves dont la plus ancienne peut être trouvée dans un euchologion glagolitique du $\mathrm{XI}^{\mathrm{e}}$ siècle, où le saint apôtre Sisini (il s'agit apparemment de Sisinnios) se dresse contre les douze (cette fois) filles d'Hérode, qui sont des démons des fièvres et des troubles mentaux ${ }^{46}$. Le texte est basé sur des motifs et thèmes similaires à celui du Testament de Salomon ${ }^{47}$ et à d'autres récits de la même famille, comme par exemple les historiolae de Gylou. On note néanmoins des différences : l'évènement a lieu au Mont Sinaï, le Bien est représenté par Saint Sisinnios et l'ange Sikhail (ou Sichaël) et le Mal par les fièvres-filles d'Hérode. Le texte est devenu très populaire dans le monde slave, surtout russe, et apparaît dans de nombreux zagovor, incantations prophylactiques. Dans une version en russe mais dont l'auteur présumé est le prêtre bulgare Jérémie, membre du mouvement chrétien hétérodoxe du bogomilisme ${ }^{48}$, nous lisons : 
Près de la mer Rouge se dresse une colonne de pierre, sur la colonne est assis le saint apôtre Sisini, il regarde la mer, elle se soulève jusqu'aux cieux et d'elle sortent douze femmes aux longs cheveux. Elles disent : « Nous sommes les tresavice, filles du tsar Hérode ». Saint Sisini leur demande: «Maudits démons pourquoi êtes-vous venus ici ?» Elles lui répondent: « Nous sommes venues pour tourmenter le genre humain. Celui qui s'enivre nous nous jetons sur lui et nous le tourmentons; celui qui dort à matines, celui qui ne prie pas Dieu, qui n'observe pas les fêtes, qui boit et mange en se levant, celui-là est à nous ". Saint Sisini prie Dieu: "Seigneur, Seigneur, sauve le genre humain de ces démons maudits ». Et le Christ lui envoya deux anges Sichaël et Anos, et les quatre évangélistes, et ils se mirent à frapper les tresavice avec des bâtons de fer, leur donnant trois mille coups par jour. Les tresavice les priaient disant: «Ne nous battez pas là où nous entendrons vos noms saints, dans les familles où l'on honore votre nom nous fuirons à la distance de trois journées ». Et saint Sisini leur demanda : "Quels sont vos noms diaboliques? " La première dit Treseja (la trembleuse), la seconde Ognieja (la fièvre ardente), « car de même que la flamme du poêle brûle le bois poissé, de même la fièvre ardente brûle le corps de l'homme ». La troisième dit: "Mon nom est Lideja (la glacée, le frisson) ». La Lideja fait trembler le corps de l'homme comme une glace froide et l'homme ne peut pas même se réchauffer dans un poêle. La quatrième dit: "Je m'appelle Gnieteja (l'oppression) ». Gnieteja pèse sur les flancs de l'homme et il ne peut plus manger. La cinquième dit : «Je m'appelle Ginousa »; elle pèse sur la tête de l'homme; elle lui brise les épaules et elle se manifeste par la toux. La sixième dit: «Je m'appelle Gloukheja (la sourde)». La Gloukheja tombe sur la tête de l'homme, lui bouche les oreilles, lui brise la tête. C'est par elle que l'homme devient sourd. La septième dit : «Je m'appelle Lomeja (la brisante) ». Lomeja brise les os et le cerveau de l'homme, comme un grand vent brise le bois sec. La huitième dit: «Je m'appelle Puchneja (l'enfleuse)». C'est elle qui en soufflant fait pousser les tumeurs. La neuvième dit: "Je m'appelle Jelieja (la jaune)». La jaunisse est comme une fleur jaune dans un champ. La dixième dit: "Je m'appelle Kerkoussa (la déchireuse)". C'est la pire de toutes, elle déchire les veines des mains et des pieds. La onzième dit : «Je m'appelle Gledeja ». Celle-là aussi est la pire de toutes : dans la nuit elle ne laisse pas de sommeil à l'homme et les démons fondent sur cet homme et sa raison s'égare. La douzième dit : « Je m'appelle Neveja (j'ignore le sens de ce mot)». C'est la plus âgée de toutes, la danseuse, celle qui coupa la tête de saint Jean. C'est la pire de toutes, quand elle saisit un homme il faut que cet homme meure ${ }^{49}$.

17 En analysant le texte, nous constatons que les démons qui apparaissent sous la forme de courants d'air ou de tornades sont représentés par douze (selon certains manuscrits sept seulement ${ }^{50}$ ) femmes ayant les cheveux lâchés, caractéristique typique des femmes démoniaques. Ensuite, les femmes se font passer pour les filles du tsar Hérode - le terme tsar, " roi », est adapté au contexte russe, en tant que symbole du tortionnaire du genre humain. Pour exterminer, ou du moins apprivoiser les démons, le Saint demande l'intervention divine, plus précisément de Sichaël et d'Anos. Devant eux, les démons sont faibles, implorent la miséricorde du Saint, et confessent leurs noms. Les saints et les anges se mettent à frapper les tresavice ${ }^{51}$ avec des bâtons, leur donnant trois mille coups par jour ${ }^{52}$. En outre, tous les démons donnent leurs noms, tous liés aux symptômes de la fièvre. Enfin, le douzième démon, le plus dangereux, qui s'appelle Neveja, est Salomé elle-même.

18 Comme on l'a déjà signalé, les deux historiolae, le roumain et le russe, remontent aux récits grecs de Gylo dont l'origine est probablement assez ancienne, probablement pas grecque mais orientale ${ }^{53}$. Il est possible néanmoins que cette tradition bien connue en Europe de l'Est liant Hérodiade et Jean le Baptiste ${ }^{54}$ à la fièvre n'ait pas été inconnue à 
l'Europe de l'Ouest. Dans un poème de 1496, intitulé La vie saint Jehan Baptiste, la fille tombe malade après la décapitation :

Et la fille par grant present

Le chief presenta a sa mere.

Mais il advint lors en present

A la mere douleur amere.

Car oncques puis ne fut journee,

Que ne tumbast troys foys le iour:

En tous les iours fut tourmentee

En maladie et en doulour.

Nous devons tel saint reclamer

Qui de tel doulour et tel peine

Nous peut trestout bien preserver

De maladie si villaine ${ }^{55}$.

À propos de ce poème, Auguste Breuil rapporte que, quelle que soit l'origine de la croyance qui a fait attribuer au précurseur la guérison de l'épilepsie, la source témoigne que cette maladie, étroitement liée à la fièvre, portait le nom de mal de Saint Jean vers la fin du XIV siècle $^{56}$. Il mentionne par ailleurs une superstition généralement répandue en France, selon laquelle «le premier seau d'eau tiré d'un puits lorsque minuit sonne dans la nuit de la St-Jean a la vertu de guérir la fièvre ${ }^{57} »$. Ambroise Paré confirme qu'on nomme l'épilepsie le mal Saint Jean, « pource que la teste de Saint Jean cheut en terre lorsqu'il fut décapitée, puis posée dedans un plat à l'appétit d'Hérodias ${ }^{58}$ ». Selon Jean-Paul Amann, l'origine des expressions «mal de Saint-Jean » et «mal de saint» est complexe: ils doivent probablement leur existence au basculement violent de la tête de Saint Jean lors de sa décapitation, ou au fait que Saint Jean souffrait aussi des crises épileptiques ${ }^{59}$. Enfin, ce lien entre la maladie et le Saint se manifeste dans le calendrier populaire : l'épilepsie s'appelle morbus solsticialis, et la fête de Saint Jean est célébrée le jour du solstice d'été ${ }^{60}$.

\section{Le lac d'Hérodiade}

Il ressort clairement de ce qui précède que la représentation d'Hérodiade en tant que personnification de la fièvre est un lieu commun dans les sources d'Europe orientale, principalement slaves, sans que cela soit la règle. En Grèce moderne, selon une croyance :

À la fête de Saint Jean, les gens ne mangent ni raisins noirs ni figues noires; et ce, parce que ces fruits doivent leur couleur au sang du saint décapité. Après avoir prêché le sermon du Christ, Saint Jean arriva au pays d'Hérode. Salomé dansait fort bien. Sa mère voulait que Saint Jean épouse Salomé, mais celui-ci refusa catégoriquement. Salomé dansa si bien un soir que son père lui promit en récompense de lui offrir ce qu'elle voudrait. Elle répondit : «je vais demander à ma mère; elle me le dira ». Sa mère dit à Salomé de demander la tête de Saint Jean. Hérode ne souhaitait pas le tuer mais il avait donné sa parole et ne pouvait revenir sur une promesse. Il envoya alors le bourreau décapiter Saint-Jean. Salomé voulut voir tout cela. Quand elle arriva, le Saint était en train de mourir et tressaillait encore. Quand elle vit la tête du Saint, elle fut tout de suite prise d'une grande fièvre. C'est depuis Salomé que l'on connait la fièvre. Avant elle, cela n'existait pas $^{61}$.

Cependant, la croyance précédente n'est pas la seule croyance grecque moderne concernant Hérodiade. Selon une autre, elle est punie éternellement dans une rivière : 
Hérodiade, qui demanda la décapitation de Saint Jean, voulut une fois traverser une rivière gelée. Mais dès qu'elle posa ses pieds maudits sur la rivière, la glace brisa et elle tomba dans un trou. Uniquement sa tête resta en dehors de la glace afin qu'elle soit punie éternellement pour la mort de Saint Jean ${ }^{62}$.

Il est certain que cette dernière croyance est assez ancienne ${ }^{63}$ et fait déjà l'objet d'une lettre apocryphe d'Hérode à Pilate écrite entre le $\mathrm{v}^{\mathrm{e}}$ et le $\mathrm{VII}^{\mathrm{e}}$ siècle en syriaque et en grec $^{64}$, dans laquelle Hérode exprime sa tristesse devant la mort de sa fille :

Je suis dans une grande anxiété. Je t'écris ces choses, afin que lorsque tu les auras entendues tu t'affliges sur mon sort. Comme ma fille Hérodiade, qui m'était chère, jouait sur un étang profond dont la surface était gelée, voici que la glace se brise sous ses pas, son corps s'enfonce, sa tête est détachée du tronc et reste sur la surface de la glace. Et, hélas! la mère tient la tête ensevelie dans son giron et toute ma maison est dans un grand deuil ${ }^{65}$.

Beaucoup plus tard, le moine et historien byzantin du XIII ${ }^{e}$ XIV ${ }^{e}$ siècle, Nicéphore Calliste Xanthopoulos, reprenant cette croyance, note dans son Histoire ecclésiastique que la fille d'Hérodiade

s'était ingérée de faire quelque voyage en temps d'hiver et en son chemin y avait une rivière à passer. Et parce que la gelée l'avait si bien fait prendre et coller ensemble, que l'on n'y voyait sinon une glace continuelle : pour la passer plus à son aise, elle se met à pied : mais ainsi qu'elle était dessus, la glace va rompre (et ce par l'ordonnance divine) tellement qu'elle tombe dans l'eau jusqu'au col: et remuant les parties basses de son corps, elle balle doucement, non sur terre, mais dedans l'eau: et la méchante tête, gelée par la force de la froidure et la glace, et avec ce blessée et séparée du reste du corps, non avec ferrement ou glaive : mais avec des croûtes d'eau glacée, représente un spectacle de danse mortelle, par-dessus les glaçons, qui rafraichissait aux regardants la mémoire de ce qu'elle avait fait ${ }^{66}$.

Il est possible que cette relation étroite d'Hérodiade avec un lac ou une rivière ne soit pas sans incidence sur le fait que les filles démoniaques d'Hérode, dans les textes slaves, sortent de l'eau. Après tout, dans un sortilège letton, qui semble inspiré des historiolae russes sur les filles d'Hérode, les jeunes filles démoniaques et dansantes vivent dans une rivière gelée :

Va, fièvre lâche, à un pont enjambant une grande rivière, baisse les yeux : dans la rivière, il y a cinq jeunes filles rouges qui dansent sur des morceaux de glace; là tu as regardé, là tu restes! Les jeunes filles disparaissent, les morceaux de glace fondent, la fièvre lâche disparaît. Notre père $[. . .]^{67}$.

Il n'y a aucun doute sur l'existence d'une relation entre Hérodiade, l'eau (sous forme de rivière ou lac) et la fièvre. Mais quelle pourrait être la raison la plus profonde de l'existence d'une telle relation? Pour répondre à cette question, nous devrons nous appuyer sur la théorie d'Alphons Barb sur les démons de la fièvre et leur nature aquatique comme elle est formulée dans son article sur le démon Antaura ${ }^{68}$. Le spécialiste analyse l'inscription grecque suivante dans un petit parchemin trouvé dans un tombeau romain du III siècle à Carnuntum en Autrice :

Contre la migraine (ou, si nous traduisons littéralement, « le demi-mal de tête »)

Antaura [c'est évidemment le nom du démon de la maladie] est sortie de la mer. Elle

a crié à haute voix, elle a crié comme une vache. Artémis d'Éphèse vient à sa rencontre. « Antaura, où amènes-tu la migraine ? " ${ }^{69}$

Le reste du parchemin est abîmé. Les mêmes éléments sont répétés dans un manuscrit grec médiéval de l'Italie du Sud. Les différences ne sont pas nombreuses: Antaura est remplacé par le nom Migraine, et Artémis d'Éphèse par Jésus. Jésus rugit, le Seigneur Jésus-Christ vient à sa rencontre et lui dit : 
Où apportes-tu le mal de tête et la migraine et la douleur dans le crâne et dans les yeux et l'inflammation et les larmes et le leucome et les vertiges? Et le mal de tête a répondu à notre Seigneur Jésus-Christ : « Nous [notons que le pluriel, le mal de tête est accompagné de toute sa famille impie de maux !] allons-nous asseoir dans la tête du serviteur de Dieu... et ainsi de suite. $»^{70}$

Nous remarquons alors que le démon qui s'assoit sur la tête n'est pas seul mais, comme dans le cas d'Hérodiade, accompagné de ses sœurs acolytes. Cette deuxième version est de toute évidence la forme chrétienne d'une tradition et d'un texte païen, comme la référence à Artémis le prouve. Le démon qui vient de la mer, comme les filles d'Hérode examinées plus haut, est, dans le premier texte, Antaura, nom composé d'anti (contre) et aura (vent doux) ${ }^{71}$. Selon l'explication de Barb, ce mal de tête-démon appelé dans l'amulette de Carnuntum « Antaura » est ce vent néfaste et oppressant que l'on connaît pour provoquer des migraines et des affections similaires, venant généralement de la mer (comme le sirocco italien) «avec des bruits sauvages appropriés ${ }^{72}$ ». Il faut noter d'ailleurs que l'aura signifiait aussi l'épilepsie, maladie, comme nous l'avons vu, étroitement liée à la fièvre.

Si nous nous rappelons maintenant qu'Hérodiade est punie par le souffle de la tête de Jean le Baptiste et qu'elle a été condamnée dans une danse aérienne, alors nous pouvons supposer que l'Hérodiade des croyances populaires a une substance éthérée. Cette possibilité explique probablement sa relation à la fois à la fièvre et à l'eau, source d'aura, qui semble prédominer dans les traditions de l'Europe orientale.

Quoi qu'il en soit, ce qui n'est pas discutable c'est que la figure biblique a acquis dans la conscience populaire des dimensions non issues des Écritures ou des textes ecclésiastiques, mais d'un contexte apocryphe ou mythologique. Passant dans le surnaturel, Hérodiade s'est éloignée du contexte historique et a cessé d'être uniquement la cause de la mort de Jean le Baptiste. Elle a été transformée en une déesse païenne et démoniaque, souveraine de sorcières, une fée, un génie et a été liée dans des récits apocryphes ou folkloriques à la fièvre et l'eau. Et, bien que les artistes occidentaux puissent davantage s'inspirer du mythe biblique que de celui forgé par l'imagination populaire, faisant de Hérodiade une sorcière ou un génie, cependant, le mouvement néopaganiste, la fameuse Wicca, combinant croyances d'origines différentes (comme par exemple le druidisme et le chamanisme), a vu en Hérodiade une déesse à vénérer. Plus précisément, en 1899, le folkloriste amateur américain Charles Leland a publié son livre Aradia, ou l'évangile des sorcières ${ }^{73}$, qui réunit une multitude de croyances et de pratiques magiques de Toscane. Dans les quinze chapitres examinant les survivances du paganisme en Italie, la figure centrale est Aradia (Hérodiade) ayant des rapports évidents à la sorcellerie. Bien que le livre contienne de nombreuses informations inexactes et fallacieuses, il a été largement lu par les néopaïens et a profondément influencé la composition et les idées principales du mouvement. Ainsi, par le biais de Wicca, Hérodiade a été réactivée et placée à côté d'un ensemble d'anciennes divinités telles que Gaïa, Diana ou Hécate. La distance entre la version originale - biblique - d'Hérodiade et ses versions ultérieures augmente avec la multiplication des altérations. Mais qu'elle soit altérée ou polymorphe, cette figure féminine - symbole intemporel de libertinage, traitée par Éliphas Lévi comme mère des «folles danseuses des bals publics ${ }^{74} »-$ reste bien vivante pour la conscience collective. 


\section{BIBLIOGRAPHIE}

\section{Bibliographie}

ABRy Christian \& JoISTEN Alice, « Le Roi Hérode, chasseur sauvage en Savoie et Dauphiné », Le Monde alpin et rhodanien. Revue régionale d'ethnologie, $\mathrm{n}^{\circ}$ 4, 2001.

AlBERT LE GRAND, Summa theologica (II,31).

AmANN Jean-Paul, Épilepsie, connaissance du cerveau et société, Québec, Les Presses de l'Université Laval, 2006.

BARB Alphons, « Antaura. The Mermaid and the Devil's Grandmother: A Lecture », Journal of the Warburg and Courtauld Institutes, $\mathrm{n}^{\circ} 29,1966$.

Bochet Marc, Salomé. Du voilé au dévoilé. Métamorphoses littéraires et artistiques d'une figure biblique, Paris, Cerf, 2007.

BORMANS Jean H., Notae in Reinardum vulpem, Gand, Annoot-Braeckman, 1836.

BREUIL Auguste, « Du culte de saint Jean-Baptiste et des usages profanes qui s'y rattachent », Mémoires de la Société des Antiquaires de Picardie, nº 8, Amiens, Duval et Herment, 1845, p. 155-244.

ClAUDEL Paul-André, Salomé. Destinées imaginaires d’une figure biblique, Paris, Ellipses, 2013.

COLLIN DE PLANCY Jacques, Dictionnaire Infernal [1818], Bruxelles, Chez Tous Les Libraires, 1845, p. 89.

CONYBEARE Frederick (éd.), « The Testament of Solomon », The Jewish Quarterly Review, n 11, 1898.

DARST David H., « Witchcraft in Spain: The Testimony of Martin de Castanega's "Treatise on Superstition and Witchcraft" (1529) ", Proceedings of the American Philosophical Society, $\mathrm{n}^{\circ}$ 123/5, 1979.

DOTTIN-ORSINI Mireille, Salomé, Paris, Autrement, 1996.

ELIADE Mircea, « Notes on the Călușari », Journal of the Ancient Near Eastern Society of Columbia University, $\mathrm{n}^{\circ} 5,1973$.

ELIADE Mircea, « Some Observations on European Witchcraft », History of Religions, n 14/3, 1975.

FILOTAS Bernadette, Pagan Survivals, Superstitions and Popular Cultures, Toronto, PIMS, 2005.

Gauchat Louis, Glossaire des Patois de la Suisse Romande, Genève, Droz, 1978, p. 1011.

GAUTHIER Claudine, Saint Jean et Salomé. Anthropologie du banquet d'Hérode, Tours, Éditions Lume, 2008.

GinZBURG Carlo, Le Sabbat des sorcières, trad. M. Aymard, Paris, Gallimard, 1989, p. 141

GINZBURG Carlo, Ecstasies. Deciphering with the Witches' Sabbath, Londres/Sydney/Auckland/

Johannesburg, Hutchinson Radius, 1990.

GREENFIELD Richard, «Saint Sisinnios, the Archangel Michael and the Female Demon Gylou: the

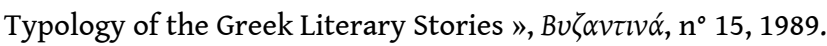

GRIMM Jacob, Teutonic Mythology, Londres, W. Swan Sonnenschein \& Allen, 1880, vol. I. 
HUTTON Ronald, Triumph of the Moon, Oxford, Oxford University Press, 2000.

KENCIS Toms, « Salome on ice: A case of a rare Latvian fever charm », Incantatio. An International Journal on Charms, Charmers and Charming, $\mathrm{n}^{\circ}$ 5, 2017, p. 29-35.

KLIGMAN Gail, Căluș: Symbolic Transformation in Romanian Ritual, Chicago, Chicago University Press, 1977.

KLoss Waldemar, « Herodias the Wild Huntress in the Legend of the Middle Ages [Part 1] », Modern Language Notes, $n^{\circ} 23 / 3,1908$, p. 82-85.

KLoss Waldemar, " Herodias the Wild Huntress in the Legend of the Middle Ages [Part 2] », Modern Language Notes, $\mathrm{n}^{\circ} 23 / 4,1908$, p. 100-102.

KoKKINOS Nikos, The Herodian Dynasty: Origins, Role in Society and Eclipse, Sheffield, Sheffield Academic Press, 1998.

Koopmans Jelle, Le Théâtre des exclus au Moyen Âge. Hérétiques, sorcières et marginaux, Paris, Imago, 1997.

KRAUSE Virginia, Witchcraft, Demonology, and Confession in Early Modern France, New York, Cambridge University Press, 2015.

LEA Henri Charles, Materials Toward a History of Witchcraft, New York, T. Yoseloff, 1957.

LeCouteux Claude, Les nains et les elfes au Moyen Âge, Paris, Imago, 1998.

LeCouteux Claude, Chasses infernales et Cohortes de la nuit au Moyen Âge, Paris, Imago, 1999.

LECoUteux Claude, «Lamia ou les métamorphoses d'un croquemitaine féminin au Moyen Âge », dans Kaniskion Philias, Mélanges Michel Saunier, éd. E. Moser-Karagiannis, Athènes, 2003.

LEEK Thomas, « Holda: Between Folklore and Linguistics », Indogermanische Forschungen, $\mathrm{n}^{\circ} 113$, 2008.

LÉGER Louis, «La Bulgarie au Moyen Âge », Revue des cours littéraires de la France et de l'étranger, $n^{\circ} 36$, août 1869 , p. 573-576.

LÉGER Louis, La Bulgarie, Paris, Cerf, 1885.

LELAND Charles, Aradia or, the Gospel of the Witches, Londres, D. Nutt, 1899.

LESOURD Dominique, « Diane et les sorcières. Études sur les survivances de Diana dans les langues romanes ", Anagrom, n 1, 1972.

LEVENE Paul, A corpus of magic bowls: Incantation Texts in Jewish Aramaic from Late Antiquity, Londres, Kegan Paul, 2003.

LÉVI Éliphas, Paris dansant, ou Les filles d'Hérodiade, folles danseuses des bals publics : le bal Mabille, la Grande-Chaumière, le Ranelagh, etc., Paris, J. Breauté, 1845.

LIST Edgar A., « Holda and the Venusberg », The Journal of American Folklore, n 73/290, 1960.

MAGLIocco Sabina, « Who Was Aradia? The History and Development of a Legend ", Pomegranate: The Journal of Pagan Studies, $\mathrm{n}^{\circ}$ 18, 2002.

MAGLIocco Sabina, « Aradia in Sardinia: The Archaeology of a Folk Character », dans D. Evans et D. Green (éds), Ten Years of Triumph of the Moon, LaVergne, Hidden Publishing, 2009, p. 40-60.

MARCHAL Bertrand, Salomé entre vers et prose - Baudelaire, Mallarmé, Flaubert, Huysmans, Paris, José Corti, 2005. 
MONTFAUCON Bernard de, Supplément au Livre de l'antiquité expliquée et représentée en figures, Paris, Chez Giffart, 1724, t. I.

MoRmAndo Franco, The Preacher's Demons: Bernardino of Siena and the Social Underworld of Early Renaissance Italy, Chicago, University of Chicago Press, 1999.

Motz Lotte, « The Winter Goddess: Percht, Holda, and Related Figures », Folklore, n 95, 1984.

Mотz Lotte, The beauty and the hag: female figures of Germanic faith and myth, Vienne, Fassbaender, 1993.

Mouskes Philippe, Chronique rimée, Bruxelles, Hayez, 1838, t. II.

NisARD Désiré, Macrobe, Varron et Pomponius Méla, Paris, Dubochet, Le Chevalier, 1850.

NIVARD, Le Roman d'Ysengrin, traduit et commenté par É. Charbonnier, Paris, Les Belles Lettres, 1991.

ORgane Atsuko, " Parcours du mythe d'Hérodias : Ysengrimus, Atta Troll, Trois contes, Salomé », Romantisme, $\mathrm{n}^{\circ}$ 154, 2011/4.

PARÉ Ambroise, CEuvres complètes d'Ambroise Paré revues et collationnées par J.-F. Malgaigne, Paris, Chez J.-B. Baillière, 1840, vol. 2.

PATERA Maria, "Gylou, démon et sorcière du monde byzantin au monde néogrec », Revue des études byzantines, $\mathrm{n}^{\circ}$ 64-65, 2006.

PATERA Maria, « Exorcismes et phylactères byzantins : écrire, énoncer les noms du démon », Cahiers Mondes anciens, $\mathrm{n}^{\circ} 1,2010$.

PICO DELla MIRANDOLA Giovanni Francesco, Libro detto strega o delle illusioni del demonio, Bologne, Beneditti, 1524, p. 16.

Politis Nikolaos G., Meletai peri tou viou kai tis glossis tou ellinikou laou : Paradosis, Athènes, Tipis P. D. Sakellariou, 1904.

RUMPF Marianne, Perchten: populäre Glaubensgestalten zwischen Mythos und Katechese, Wurtzbourg, Königshausen \& Neumann, 1991.

RUSSELL Jeffrey Burton, Witchcraft in the Middle Ages, Ithaca/Londres, Cornell University Press, 1972.

RYAN William F., The bathhouse at midnight: an historical survey of magic and divination in Russia, Pennsylvanie, Pennsylvania State University Press, 1999.

RYAN William F., « Ancient Demons and Russian Fevers », dans C. Burnett et W. F. Ryan (éds), Magic and the Classical Tradition, Londres, Warburg Institute, 2005, p. 37-58.

SCHWENTZel Christian-Georges, Hérode le Grand, Paris, Pygmalion, 2011.

SÉBILLOT Paul, Le folk-lore de France : Le ciel et la terre.- v.2. La mer et les eaux douces.- t.3. La faune et la flore.- t.4, Paris, Librairie orientale \& américaine, 1904.

SMITH Susan L., The Power of Women: A Topos in Medieval Art and Literature, Philadelphie, University of Pennsylvania Press, 1995.

TiмотіN Emanuela, «Queen of the fairies and biblical queen. Notes on the Romanian Herodias ", Acta Ethnographica Hungarica, $n^{\circ}$ 54/2, 2009, p. 365-378. 
Tiмotin Emanuela, «Irodia, doamna zânelor. Notes sur les fées roumaines et leur cohorte fantastique », dans K. Ueltschi (éd.), Les entre-mondes. Les vivants, les morts, Paris, Klincksieck, 2009, p. 179-204.

TOPORKOv Andreï Lvovitch, « Arhangel Mihail pobivaet trjasovic : genezis, istorija i social'noe funkcionirovanie ", In Umbra: Demonologija kak semioticheskaja sistema. Al'manah. Vyp. 1. Moscou, RGGU, 2012.

TOPORKOv Andreï Lvovitch, « Sisinieva molitva ot trjasovic i drevnerusskie indeksy lozhnyh knig », Zapretnoe / dopuskaemoe / predpisannoe v fol'klore, Moscou, Izdatel'skij centr RGGU, 2013.

TRocmé Étienne, L'évangile selon saint Marc, Paris, Labor et Fides, 2000.

TURDEANU Émile, « Notes de mythologie roumaine : 2. Artémis-Diane et les fées roumaines », dans E. Turdeanu (éd.), Studii şi articole literare, Bucarest, Minerva, p. 53-57.

VAN TIL Salomon, Disputatio theologico-chronologica de Johannis Baptistae incarceratione fictitia, Herodiana vincula antecedente, Leyde, Elzevier, 1710.

VARIOT Joseph, Étude sur l'histoire littéraire, la forme primitive et les transformations des Évangiles apocryphes, Paris, Ernest Thorin, 1878.

WIER Jean, Histoires, disputes et discours des illusions et impostures des diables, des magiciens infâmes..., livre III, Paris, A. Delahaye et E. Lecrosnier, 1885.

WoLfF Oskar, Altfranzösische Volkslieder, Leipzig, Fleischer, 1831.

WRONA Carole, Imaginaires de la taille humaine au cinéma : de la figuration du nain, Paris, L'Harmattan, 2006.

XANTHOPOULOS Nicéphore Calliste, L'Histoire ecclésiastique, Paris, Sébastien Nivelle, 1577.

Zochios Stamatios, « Lamia: A Sorceress, a Fairy or a Revenant? », Tric Trac. Journal of World Mythology and Folklore, $\mathrm{n}^{\circ}$ 4, 2013.

\section{NOTES}

1. Pour une image plus complète sur cette figure biblique et ses représentations artistiques, voir M. Dottin-Orsini, Salomé, 1996 ; P.-A. Claudel, Salomé. Destinées imaginaires d'une figure biblique, 2013 ; B. Marchal, Salomé entre vers et prose - Baudelaire, Mallarmé, Flaubert, Huysmans, 2005 ; A. Organe, «Parcours du mythe d'Hérodias : Ysengrimus, Atta Troll, Trois contes, Salomé », 2011, p. 149-160 ; C. Gauthier, Saint Jean et Salomé. Anthropologie du banquet d'Hérode, 2008 ; M. Bochet, Salomé. Du voilé au dévoilé. Métamorphoses littéraires et artistiques d'une figure biblique, 2007.

2. S. Smith, The Power of Women: A Topos in Medieval Art and Literature, 1995.

3. Les sources primaires proviennent de Flavius Josèphe (AJ XVIII, 137). Voir aussi C.G. Schwentzel, Hérode le Grand, 2011, p. 225 ; N. Kokkinos, The Herodian Dynasty: Origins, Role in Society and Eclipse, 1998, p. 264-268.

4. On peut supposer que Salomé est née à Rome mais on ne connaît que très peu de choses sur sa vie : ni le lieu de naissance, ni ses dates de naissance et de décès. La seule référence explicite à elle se fait dans le livre XVIII des Antiquités judaïques de Flavius Josèphe. Voir M. Bochet, ouvr. cité, p. 15.

5. É. Trocmé, L'évangile selon saint Marc, 2000, p. 175.

6. W. Kloss, « Herodias the Wild Huntress in the Legend of the Middle Ages [Part 1]», 1908, p. 85.

7. M. Dottin-Orsini, ouvr. cité, p. 15. 
8. Cité par J. Grimm, Teutonic Mythology, 1880, p. 283-284.

9. Pour plus d'informations sur Diane et ses diverses formations au sein des croyances populaires européennes, voir D. Lesourd, «Diane et les sorcières. Études sur les survivances de Diana dans les langues romanes », 1972, p. 55-74 ; B. Filotas, Pagan Survivals, Superstitions and Popular Cultures, 2005, p. 114-117.

10. Le mot jeu(x) (ailleurs ludus) signifie dans ce cas le sabbat.

11. Cité par A. Breuil, « Du culte de saint Jean-Baptiste et des usages profanes qui s'y rattachent ", 1845, p. 181-182. Voir aussi J. Koopmans, Le Théâtre des exclus au Moyen Âge. Hérétiques, sorcières et marginaux, 1997, p. 119.

12. Albert le Grand, Summa theologica (II,31).

13. D. Darst, «Witchcraft in Spain: The Testimony of Martin de Castanega's "Treatise on Superstition and Witchcraft" (1529)», 1979, p. 306.

14. H. C. Lea, Materials Toward a History of Witchcraft, 1957, p. 406.

15. J. Wier, Histoires, disputes et discours des illusions et impostures des diables, des magiciens infâmes..., livre III, 1885, p. 318.

16. G. F. Pico Della Mirandola, Libro detto strega o delle illusioni del demonio, 1524, p. 16.

17. C. Ginzburg, Ecstasies. Deciphering with the Witches' Sabbath, 1990, p. 92.

18. Voir F. Mormando, The Preacher's Demons: Bernardino of Siena and the Social Underworld of Early Renaissance Italy, 1999, p. 268.

19. S. Van Til, Disputatio theologico-chronologica de Johannis Baptistae incarceratione fictitia, Herodiana vincula antecedente, 1710.

20. (Frau) Percht, Bercht, Perchta, Berchta, etc. et (Frau) Holda, Hulda, Holle, etc. sont deux figures principales de la mythologie germanique, deux dames de la nuit, manifestant un caractère maléfique mais assez souvent bénéfique aussi. La bibliographie qui les concerne est très extensive : L. Motz, « The Winter Goddess: Percht, Holda, and Related Figures », 1984, p. 151-166 ; L. Motz, The beauty and the hag: female figures of Germanic faith and myth, 1993 ; M. Rumpf, Perchten: populäre Glaubensgestalten zwischen Mythos und Katechese, 1991 ; T. Leek, « Holda: Between Folklore and Linguistics », 2008, p. 312-338 ; E. List, « Holda and the Venusberg », 1960, p. 307-311.

21. L. Motz, ouvr. cité, p. 124.

22. Cité par B. de Montfaucon, Supplément au Livre de l'antiquité expliquée et représentée en figures, 1724 , t. I, p. 312.

23. J. Collin de Plancy, Dictionnaire Infernal [1818], 1845, p. 89.

24. Polycraticus (II,17). Cité par C. Lecouteux, Chasses infernales et Cohortes de la nuit au Moyen Âge, 1999 , p. 17.

25. Comme Claude Lecouteux le rapporte, les canonistes et les clercs du Moyen Âge se réfèrent à une coutume, parfois évoquée en deux mots - mensas ornare - selon laquelle on dépose des offrandes ou des cadeaux sur la table dressée (C. Lecouteux, ouvr. cité, p. 21).

26. D. Nisard, Macrobe, Varron et Pomponius Méla, 1850, p. 451.

27. W. Kloss, «Herodias the Wild Huntress in the Legend of the Middle Ages [Part 2]», 1908, p. 101.

28. B. de Montfaucon, ouvr. cité, p. 115.

29. Voir par exemple les traités démonologiques de Molitor, De Lamiis et Pythonicis Mulieribus (1489) et de Weyer, De Lamiis (1577) (V. Krause, Witchcraft, Demonology, and Confession in Early Modern France, 2015, p. 62). Pour plus d'informations sur cette princesse monstrueuse, voir C. Lecouteux, "Lamia ou les métamorphoses d'un croquemitaine féminin au Moyen Âge ", dans Kaniskion Philias, Mélanges Michel Saunier, 2003, p. 51-61 ; S. Zochios, « Lamia: A Sorceress, a Fairy or a Revenant?», 2013, p. 96-112.

30. Nivard, Le Roman d'Ysengrin, 1991, p. 5.

31. Le Sabbat des sorcières, 1989, p. 141.

32. J. B. Russell, Witchcraft in the Middle Ages, 1972, p. 49. 
33. J. H. Bormans, Notae in Reinardum vulpem, 1836, p. 96 ; P. Mouskes, Chronique rimée, 1838, t. II, p. cxl.

34. Cité par C. Lecouteux, ouvr. cité, 1999, p. 16.

35. Ce rapprochement entre génie et Hérodiade n'est qu'en apparence paradoxal. Dans le folklore suisse, et plus précisément dans le Jura bernois, "ils s'appellent duses ou hairodes, ce dernier terme est sans doute la déformation d'Hérodiade qui est, selon les clercs du Moyen Âge, le nom d'une des meneuses des cohortes de la nuit » (C. Lecouteux, Les nains et les elfes au Moyen Âge, 1998, p. 170). Cette identification entre Hérodiade et dusius n'est pas seulement indiquée par Claude Lecouteux. Le Glossaire des Patois de la Suisse Romande explique l'entrée «Dussats » ainsi : "Êtres surnaturels appelés autrement hairodes. Ces Duses ou Hairodes... que le peuple appelait les Dussats, Mussats ou Hairodes " (L. Gauchat, Glossaire des Patois de la Suisse Romande, 1978, p. 1011). Paul Sébillot les considère comme des lutins et les décrit comme « de petits êtres noirs et velus, parfois assez méchants, appelés les Duses ou les Hairodes» (P. Sébillot, Le folk-lore de France: Le ciel et la terre.- v.2. La mer et les eaux douces.- t.3. La faune et la flore.- t.4, 1904, p. 456).

36. Pareillement en Suisse et en France, on parle souvent de la Rosche des Hairodes et le Glossaire des Patois de la Suisse Romande note que "ces Duses ou Hairodes n'étaient peut-être que des Tsiganes ou Bohémiens, des forgerons nomades, habitant les cavernes des Duses en été » (L. Gauchat, ouvr. cité, p. 1011). Carole Wrona les lie aux Sarrasins et aux Mauriacs : «Un être humain profondément mauvais n'échapperait donc pas à la métamorphose, punition divine. Sarrasins, Mauriacs et Hairodes, autrefois grands, forts et beaux, ont rapetissé jusqu'à la taille d'un écureuil. » (C. Wrona, Imaginaires de la taille humaine au cinéma : de la figuration du nain, 2006, p. 145).

37. S. Magliocco, « Aradia in Sardinia: The Archaeology of a Folk Character », 2009, p. 55.

38. Sur cette danse traditionnelle de fertilité, voir M. Eliade, "Notes on the Călușari », 1973, p.115-122; M. Eliade, «Some Observations on European Witchcraft», 1975, p. 149-172; G. Kligman, Căluș: Symbolic Transformation in Romanian Ritual, 1977.

39. M. Eliade, art. cité, 1975, p. 160-161.

40. Ibid.

41. Bien que les textes ne précisent pas quel Hérode est mentionné, nous pouvons supposer qu'il s'agit d'Hérode Antipas, conjoint d'Hérodias et beau-père de Salomé, ici pourtant décrit comme le père de Salomé. Il faut cependant signaler que, selon la règle générale, les croyances d'origine populaire se réfèrent à un "Roi Hérode" (construisant une ambiguïté troublante entre les personnages réels que sont Hérode le Grand et son fils Hérode Antipas) en tant que figure démoniaque. Il en est ainsi de la croyance à l'Armée du Roi Hérode en Savoie, selon laquelle le roi est un cavalier diabolique, traînant des chaînes, accompagné du bruit des grelots de son cheval, faisant partie des cavaleries infernales des Douze Jours de Noël, ou un croquemitaine pour les enfants (C. Abry \& A. Joisten, « Le Roi Hérode, chasseur sauvage en Savoie et Dauphiné », 2001, p. 7-35). Le caractère démoniaque de ce « Roi Hérode » résulte de sa responsabilité supposée dans le prétendu massacre des Innocents (Matthieu 2,16-18).

42. Un procédé narratif utilisé par les magiciens, dans leurs incantations, pour renforcer l'efficacité de leurs formules (P. Levene, A corpus of magic bowls: Incantation Texts in Jewish Aramaic from Late Antiquity, 2003, p. 17).

43. Gylou, Gello, Gillo, Gelu, etc. est un démon byzantin issu de la mythologie grecque antique. Sur ce démon féminin et les phylactères contre elle, voir M. Patera, « Exorcismes et phylactères byzantins: écrire, énoncer les noms du démon», 2010. Disponible sur <http:// journals.openedition.org/mondesanciens/139>; M. Patera, "Gylou, démon et sorcière du monde byzantin au monde néogrec ", 2006, p. 311-327; R. Greenfield, "Saint Sisinnios, the Archangel Michael and the Female Demon Gylou: the Typology of the Greek Literary Stories ", 1989, p. 83-142. 
44. E. Timotin, «Queen of the fairies and biblical queen. Notes on the Romanian Herodias », 2009, p. 366.

45. Ibid., p. 370.

46. W. F. Ryan, The bathhouse at midnight: an historical survey of magic and divination in Russia, 1999, p. 244-253.

47. F. Conybeare (éd.), « The Testament of Solomon », 1898, p. 1-45.

48. L. Léger, La Bulgarie, 1885, p. 33.

49. Ibid., p. 33-34.

50. L. Léger, « La Bulgarie au Moyen Âge », août 1869, p. 575.

51. Sur le démon de la fièvre tresavica, voir W. F. Ryan, ouvr. cité, p. 244-253 ; A. L. Toporkov, "Arhangel Mihail pobivaet trjasovic: genezis, istorija i social'noe funkcionirovanie», 2012, p. 247-289; A. L. Toporkov, "Sisinieva molitva ot trjasovic i drevnerusskie indeksy lozhnyh knig ", 2013, p. 197-227.

52. Le motif des coups comme punition du démon est récurrent dans plusieurs textes de ce type d'historiolae.

53. A. Barb, « Antaura. The Mermaid and the Devil's Grandmother: A Lecture », 1966, p. 1-23.

54. En Grèce aussi, Jean le Baptiste offre protection contre la fièvre et surtout la fièvre du

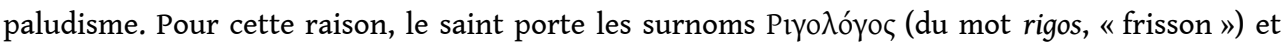

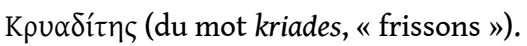

55. O. Wolff, Altfranzösische Volkslieder, 1831, p. 19.

56. A. Breuil, art. cité, p. 175.

57. Ibid., p. 237.

58. A. Paré, CEuvres complètes d'Ambroise Paré revues et collationnées par J.-F. Malgaigne, 1840, vol. 2, p. 80.

59. J.-P. Amann, Épilepsie, connaissance du cerveau et société, 2006, p. 27.

60. Ibid. Pour plus d'informations sur les rapports entre Saint Jean et l'épilepsie, voir C. Gauthier, ouvr. cité, p. 131-134.

61. Manuscrit 2392 de l'Archive du Centre de Recherches du Folklore Hellénique de l'Académie d'Athènes. Disponible sur <http://repository.kentrolaografias.gr/xmlui/handle/ 20.500.11853/292683>.

62. N. G. Politis, Meletai peri tou viou kai tis glossis tou ellinikou laou : Paradosis, 1904, p. 106.

63. L'ancien thème de la mort glacée d'Hérodiade a inspiré beaucoup plus tard Mallarmé et Apollinaire (M. Dottin-Orsini, ouvr. cité, p. 16).

64. Cité par J. Variot, Étude sur l'histoire littéraire, la forme primitive et les transformations des Évangiles apocryphes, 1878, p. 112.

65. Ibid., p. 112-113.

66. N. C. Xanthopoulos, L'Histoire ecclésiastique, 1577, p. 20-21.

67. T. Ķencis, « Salome on ice: A case of a rare Latvian fever charm », 2017, p. 31.

68. A. Barb, art. cité, p. 1-23.

69. Ibid., p. 2.

70. Ibid., p. 3.

71. Ibid.

72. Ibid.

73. C. Leland, Aradia or, the Gospel of the Witches, 1899. Pour plus d'informations, voir R. Hutton, Triumph of the Moon, 2000 ; S. Magliocco, «Who Was Aradia? The History and Development of a Legend ", 2002, p. 5-22.

74. É. Lévi, Paris dansant, ou Les filles d'Hérodiade, folles danseuses des bals publics : le bal Mabille, la Grande-Chaumière, le Ranelagh, etc., 1845. 


\section{RÉSUMÉS}

Hérodias, épouse du tétrarque de Judée Hérode Antipas, est une figure biblique connue essentiellement pour avoir demandé l'exécution de Jean le Baptiste. Sa fille Salomé, célèbre pour sa danse, apparaît sous le même nom. Mère et fille se sont transformées graduellement, dans la conscience collective des chrétiens, en un personnage diabolique sous le nom indistinct d'Hérodiade. Évacuant la perspective historique et biblique, la figure mythique d'Hérodiade a été identifiée, selon de nombreuses sources, à Diana, Bensozia, Noctiluca, aux sorcières et aux dames de la nuit, et cela dès le bas Moyen Âge. Elle était donc vénérée comme une souveraine maléfique. Parfois sous la forme d'une fée ou d'un génie du lieu, elle apparaît autant en Europe occidentale qu'orientale (comme en Roumanie, en Russie ou en Grèce) dans plusieurs pratiques, rituels, croyances et incantations liés régulièrement à la fièvre. Notre article examine le rôle multidimensionnel d'Hérodiade dans les croyances et pratiques populaires européennes. Mots en français

In the biblical context Herodias is a famous figure, wife of Herod Antipas, that demanded the execution of John the Baptist. At the same time, her daughter Salome, known for her famous dance, appears under the same name. Thus, Herodias and Salome, gradually transformed, in the collective consciousness of Christians, into a diabolical character under the indistinct name of Herodias. Leaving the historical and biblical context, the mythical figure of Herodias has been identified, as several sources testify, to Diana, Bensozia, Noctiluca, the witches and the ladies of the night, and that already since the Low Middle Age. She was therefore revered as an evil sovereign. Sometimes a fairy or a genius loci, she appears in several practices, rituals, beliefs and incantations of not only western but also eastern European origin (e.g. Romania, Russia, Greece), regularly linked to fever. This article examines this multidimensional role of Herodias in popular beliefs and practices in Europe.

\section{INDEX}

Mots-clés : Hérodiade, Salomé, Diana, sorcières, fées, génies, incantations, fièvre Keywords : Herodias, Salome, Diana, witches, fairies, spirits, incantations, fever

\section{AUTEUR}

\section{STAMATIOS ZOCHIOS}

École Pratique des Hautes Études - PSL 